Pacific Journal of Mathematics

EXTENSIONS OF AN INEQUALITY BY PÓLYA AND SCHIFFER

OR VIBRATING MEMBRA 


\section{EXTENSIONS OF AN INEQUALITY BY PÓLYA AND SCHIFFER FOR VIBRATING MEMBRANES}

\section{Catherine Bandle}

The inequality by P6lya and Schiffer considered in this paper is concerned with the sume of the $n$ first reciprocal eigenvalues of the problem $\Delta u+\lambda u=0$ in $G, u=0$ on $\partial G$. First we extend this inequality to the problem of an inhomogeneous membrane $\Delta u+\lambda \rho u=0$ in $G, u=0$ on $\partial G$. Then we prove a sharper form of it for a class of homogeneous membranes with partially free boundary. The proofs are based on a variational characterization for the eigenvalues and use conformal mapping and transplantation arguments.

The inequality by Pólya and Schiffer considered in this paper is concerned with the eigenvalue problem $\Delta \varphi+\lambda \varphi=0$ in a Jordan domain $G, \varphi=0$ on $\partial G$. It can be stated as follows: Among all domains with given maximal conformal radius $\dot{r}$, the circle yields the minimum of the expression $\sum_{i=1}^{n} \lambda_{i}^{-1}$. This theorem is related to the geometrical inequality

$$
\pi \dot{r}^{2} \leqq A
$$

where $A$ denotes the total area of $G$. The aim of this paper is (i) to extend the inequality by Pólya and Schiffer to the problem of an inhomogeneous membrane fixed on the boundary, (ii) to sharpen it for certain kinds of elastically supported, homogeneous membranes. Instead of considering the problem of an inhomogeneous membrane we will study the equivalent eigenvalue problem $L u+\lambda u=0$ where $L=\Delta / \rho$ is the Beltrami operator of an abstract surface with the line element $d s^{2}=\rho\left(d x^{2}+d y^{2}\right)$. With the help of inequalities by Alexandrow [1], we will derive first some relations between $\dot{r}, \rho$ and the Gaussian curvature of the surface. These results will be needed for the theorem concerning the eigenvalue problem. Its proof is essentially based on a method indicated by Hersch in [6] which uses conformal mapping and transplantation arguments. In the last part, we give an isoperimetric inequality for a class of plane membranes. The extremal domain is in this case the circular sector.

\section{Geometrical preliminaries.}

DEFINITIONS 1.1. Let $\Sigma$ be an abstract surface given by a Jordan domain $G$ in the $z$-parameter plane $(z=x+i y)$, and by the metric $d s^{2}=\rho(z)|d z|^{2}$ where $\rho(z)$ is an arbitrary positive function in $C^{2}$. 
$A(B)=\iint_{B} \rho d x d y$ is the area of a domain $B \cong \Sigma$ and

$$
L(\gamma)=\int_{r} \sqrt{\rho}|d z|
$$

is the length of an arc $\gamma \subseteq \Sigma$. The Gaussian curvature has the form

$$
K_{G}=\left(-\Delta_{Z} \ln \rho\right) / 2 \rho\left[\Delta_{z}=\frac{\partial^{2}}{\partial x^{2}}+\frac{\partial^{2}}{\partial y^{2}}\right] \text {. }
$$

We shall assume that the inequality $K_{G} \leqq K_{0}$ holds in $G$. Consider a surface $\mathscr{M}_{K_{0}}$ of constant curvature $K_{0}$ given in the following isothermic representation:

(i) $w$-plane $(w=u+i v)$ with the metric

$$
d s^{2}=\frac{4 c^{2}}{\left(1+|w|^{2}\right)^{2}}|d w|^{2} \quad \text { if } \quad K_{0}=1 / c^{2}
$$

(ii) interior of the unit circle $\{w ;|w|<1\}$ with the metric

$$
d s^{2}=\frac{4 c^{2}}{\left(1-|w|^{2}\right)^{2}}|d w|^{2} \quad \text { if } \quad K_{0}=-1 / c^{2} .
$$

(iii) $w$-plane with the metric $d s^{2}=|d w|^{2}$ if $K_{0}=0$.

We shall define the metric of $\mathscr{M}_{K_{0}}$ by $d s^{2}=g(w)|d w|^{2}$, where $g(w)$ depends on $K_{0}$ and is determined by one of the preceding formulas. Let $f_{a}(z)$ be the conformal mapping from $G$ onto the unit circle $\{w ;|w|<1\}$ with $f_{a}(a)=0$ and $f_{a}^{\prime}(a)>0$. The conformal radius of the point $a$ with respect to $G$ is then defined as $r_{a}(G)=1 / f_{a}^{\prime}(a)$ [9, p. 16]. We set

$$
R_{a}(G)=\left\{\begin{array}{lll}
\frac{1}{2} \sqrt{\rho(a)\left|K_{0}\right|} r_{a}(G) & \text { if } & K_{0} \neq 0 \\
\sqrt{\rho(a)} r_{a}(G) & \text { if } & K_{0}=0 .
\end{array}\right.
$$

ExAmple. If $G$ is a circle with the radius $r_{0}$, the center in the origin and $\rho(z)=g(z)$, then $R_{0}(G)=r_{0} . \quad w_{a}(z)=R_{a}(G) f_{a}(z)$ maps $G$ onto the circle $\left\{w ;|w|<R_{a}(G)\right\}$, and $z_{a}(w)$ denotes its inverse. We shall denotes the circle $\{w ;|w|<\varepsilon\}$ by $C_{\varepsilon}$. $R_{a}(G)$ has been chosen in such a way that

$$
\iint_{C_{\varepsilon}} g(w) d u d v=\iint_{z_{a}\left(C_{\varepsilon}\right)} \rho(z) d x d y+o\left(\varepsilon^{2}\right)
$$

Since

$$
\iint_{C_{\varepsilon}} g(w) d u d v=\left\{\begin{array}{lll}
4 \pi c^{2} \varepsilon^{2}+o(\varepsilon)^{2} & \text { if } \quad K_{0} \neq 0 \\
\pi \varepsilon^{2} & \text { if } \quad K_{0}=0
\end{array}\right.
$$

it follows that 


$$
\lim _{\varepsilon \rightarrow 0} \frac{1}{\varepsilon^{2}} \iint_{C_{\varepsilon}} g(w) d u d v=\lim _{\varepsilon \rightarrow 0} \frac{1}{\varepsilon^{2}} \iint_{z_{a}\left(C_{\varepsilon}\right)} \rho(z) d x d y
$$

exists and is different from zero.

1.2. Some Properties of $R_{a}(G)$.

(a) $R_{a}(G)$ is invariant under conformal mapping.

Proof. Let $\xi(z): \Sigma \Rightarrow \hat{\Sigma}$ be a conformal mapping and let $z(\xi)$ be its inverse. We set $\xi(a)=a$ and $\xi(G)=\hat{G}$. The line element of $\hat{\Sigma}$ is $d \hat{s}^{2}=\hat{\rho}(\xi)|d \xi|^{2}$ with $\hat{\rho}(\xi)=\rho(z(\xi))|d z / d \xi|^{2}$. Since $K_{G}$ is a conformal invariant, we have

$$
R_{\hat{a}}(\widehat{G})=\frac{1}{2} \sqrt{\hat{\rho}(\hat{a})\left|K_{0}\right|} r_{\hat{a}}(\widehat{G})=\frac{1}{2} \sqrt{\rho(a)\left|K_{0}\right|}\left|\frac{d z}{d \xi}\right|_{\xi=\hat{a}} r_{\hat{a}}(\hat{G}) .
$$

Because of the relation $|d z / d \xi|_{\xi=\hat{a}} r_{\hat{a}}(\hat{G})=r_{a}(G)$ [9], it follows that $R_{\hat{a}}(\hat{G})=R_{a}(G)$.

(b) If $K_{0}<0$, then $R_{a}(G)<1$ for any $a \in G$

Proof. The function $\hat{\rho}(w)=\rho\left(z_{a}(w)\right)\left|d z_{a} / d w\right|^{2}$ satisfies in

$$
C=\left\{w ;|w| \leqq R_{a}(G)\right\}
$$

the inequality $\Delta_{W} \ln \hat{\rho}(w) \geqq\left(2 / c^{2}\right) \hat{\rho}(w)$. By a theorem of Osserman [7]

$$
\hat{\rho}(w) \leqq \frac{4 c^{2} R_{a}^{2}}{\left(R_{a}^{2}-r^{2}\right)^{2}} \quad\left(r=|w|, R_{a}=R_{a}(G) \text { for any } \quad w \in C\right) .
$$

Since $\hat{\rho}(0)=4 c^{2}$, (6) implies $R_{a} \leqq 1$.

(c) Let $\mu_{\varepsilon}=\mu\left(\partial C, \partial C_{\varepsilon}\right)$ be the modulus of the annulus $D=C \backslash C_{\varepsilon} \quad\left[C=\left\{w ;|w|<R_{a}\right\}, \partial C\right.$ the boundary of $C ; C_{\varepsilon}=\{w ;|w|<$ $\varepsilon\}, \partial C_{\varepsilon}$ the boundary of $C_{\varepsilon}$ l. Let $h$ be the solution of the Dirichlet problem $\Delta h=0$ in $C \backslash C_{s}, h=0$ on $\partial C_{c}, h=1$ on $\partial C$ and let $D(h)$ denote the Dirichlet integral of $h$. Then $\mu_{s}=\{D(h)\}^{-1}$. In an analogous may we define $\mu\left(\Gamma, \Gamma_{\varepsilon}\right)$, where $\Gamma$ and $\Gamma_{\varepsilon}$ are boundaries of $G$ and $z_{a}\left(C_{\varepsilon}\right)$. Since the modulus is invariant under conformal mapping, we conclude that

$$
\mu_{\varepsilon}=\mu\left(\Gamma, \Gamma_{\varepsilon}\right)=\frac{1}{2 \pi} \ln \frac{R_{a}}{\varepsilon},
$$

and thus

$$
R_{a}=\varepsilon e^{2 \pi \mu_{\varepsilon}}=\lim _{\varepsilon \rightarrow 0} \varepsilon e^{2 \pi \mu \varepsilon}
$$

If $G$ is contained in $G^{\prime}$, then it follows from (7) and the Dirichlet principle that $R_{a}(G) \leqq R_{a}\left(G^{\prime}\right)$. 
(d) Let $A=A(G)=\iint_{G} \rho d x d y$ be the total area of $G$ with respect to the metric $d s^{2}=\rho|d z|^{2}$, and let $A_{C}=\iint_{C} g(w) d u d v$ be the total area of $C$ with respect to the metric $d s^{2}=g(w)|d w|^{2} . A_{C}$ takes the values

$$
A_{C}=\left\{\begin{array}{lll}
4 \pi c^{2} R_{a}^{2} /\left(1+R_{a}^{2}\right) & \text { if } \quad K_{0}=c^{-2} \\
4 \pi c^{2} R_{a}^{2} /\left(1-R_{a}^{2}\right) & \text { if } \quad K_{0}=-c^{-2} \\
\pi R_{a}^{2} & \text { if } \quad K_{0}=0 .
\end{array}\right.
$$

The following result is an extension of a classical theorem [9, problem $125 \mathrm{IV}]$. We have

$$
A \geqq A_{C} \cdot
$$

Equality holds in (9) if and only if $G$ is a geodesic circle on a surface of constant curvature $K_{0}$. (If $K_{0}>0$ we have to assume that $A<$ $\left.4 \pi / K_{0}.\right)$

Proof. Let $A_{1}(\varepsilon)$ and $A_{1}^{\prime}(\varepsilon)$ denote the area of $z_{a}\left(C_{\varepsilon}\right)$ and $C_{\varepsilon}$. By (7) and Corollary $2[3]$ it follows that

$$
\mu\left(\Gamma, \Gamma_{\varepsilon}\right)=\frac{1}{2 \pi} \ln \frac{R_{a}}{\varepsilon} \leqq \frac{1}{4 \pi}\left\{\ln \frac{A}{4 \pi-K_{0} A}-\ln \frac{A_{1}(\varepsilon)}{4 \pi-K_{0} A_{1}(\varepsilon)}\right\} .
$$

Equality holds only if $\Gamma$ and $\Gamma_{\varepsilon}$ are two "concentric" circles on a surface of constant curvature $K_{0}$. Suppose that $K_{0} \neq 0$. From (8) we have $A_{1}^{\prime}(\varepsilon)=4 \pi c^{2} \varepsilon^{2}+o\left(\varepsilon^{2}\right)$. Substituting this expression in (10), we obtain

$$
\frac{4 \pi c^{2} R_{a}^{2}}{A_{1}^{\prime}(\varepsilon)+o\left(\varepsilon^{2}\right)} \leqq \frac{A\left(4 \pi-K_{0} A_{1}(\varepsilon)\right)}{A_{1}(\varepsilon)\left(4 \pi-K_{0} A\right)}=\Phi(\varepsilon) .
$$

Since $\lim _{\varepsilon \rightarrow 0}\left(A_{1}^{\prime}(\varepsilon) / A_{1}(\varepsilon)\right)=1$ (cf. (3), (4)), it follows that

$$
R_{a}^{2}=\frac{A_{C}}{c^{2}\left(4 \pi-K_{0} A_{C}\right)} \leqq \lim _{\varepsilon \rightarrow 0} \frac{A_{1}^{\prime}(\varepsilon)+o\left(\varepsilon^{2}\right)}{4 \pi c^{2}} \Phi(\varepsilon)=\frac{1}{c^{2}} \frac{A}{\left(4 \pi-K_{0} A\right)} .
$$

This inequality implies $A_{C} \leqq A$. The case $K_{0}=0$ can be treated in exactly the same way and will therefore be omitted.

REMARKs. (1) Let $g_{z}(z, a)$ be the Green's function defined by $\Delta_{z} g_{z}(z, a)=-\delta_{a}(z)$ in $G, g_{z}(z, a)=0$ on $\Gamma . g_{w}(w, 0)$ is the corresponding Green's function in $C$. We shall use the following notations $G(t)=$ $\left\{z \in G ; g_{z}(z, a)>t\right\}, C(t)=\left\{w \in C ; g_{w}(w, 0)>t\right\} ; A_{z}(t)=\iint_{G(t)} \rho d x d y$ and $A_{w}(t)=\iint_{C(t)} g(w) d u d v$. By the same reasoning as before we can show 
that

$$
A_{z}(t) \geqq A_{w}(t) \text {. }
$$

Equality holds if and only if $G$ is a geodesic circle on a surface of constant curvature $K_{0}$. If $K_{0}>0$, we have, of course, to assume that $A_{z}(t)<4 \pi / K_{0}$.

(2) We define $\dot{R}(G)=\max _{a \in G} R_{a}(G)$. If $G$ is a circle of radius $r$ with the center at the origin and the metric $d s^{2}=g(w)|d w|^{2}$, then $R_{a}(G)=\left(r^{2}-|a|^{2}\right) /\left(1 \pm|a|^{2}\right) r$ [9]. In this case, $\dot{R}(G)=R_{0}(G)$. Because of (11) we have the isoperimetric inequality: Among all domains with given total area $A$ and with given $K_{0}$, the geodesic circles on a surface of constant curvature $K_{0}$ have the largest value of $\dot{R}(G)$. From (11) it follows that

$$
r_{a}^{2}(G) \leqq \frac{4 A}{\rho(a)\left(4 \pi-K_{0} A\right)}
$$

If $\rho \equiv 1$, then (13) reduces to $\pi r_{a}^{2}(G) \leqq A$.

2. Bounds for the eigenvalues of an inhomogeneous membrane. Let $\Sigma$ be an abstract surface given in an isothermic representation (cf. §1.1). We consider the following eigenvalue problem

$$
\begin{aligned}
\frac{\Delta_{z}}{\rho} \varphi(x, y)+\lambda \varphi(x, y) & =0 \text { in } G \\
\varphi & =0 \text { on } \Gamma \text { (boundary of } G \text { ). }
\end{aligned}
$$

$\Delta_{z} / \rho$ represents the Beltrami operator of $\Sigma$. Suppose that a countable number of eigenvalues $0<\lambda_{1}<\lambda_{2} \leqq \cdots$ exists. $R[v]=D(v) / \iint_{G} v^{2} \rho d x d y$ $\left[D(v)=\iint_{G} \operatorname{grad}^{2} v d x d y\right]$ is the Rayleigh quotient of Problem I. Let $L_{n}$ be an $n$-dimensional linear space of continuously differentiable functions which vanish on $\Gamma$, and let $v_{1}, \cdots, v_{n}$ be an orthogonal basis in $L_{n}$ with respect to the Dirichlet metric, i.e.,

$$
D\left(v_{i}, v_{j}\right)=\iint_{G} \operatorname{grad} v_{i} \operatorname{grad} v_{j} d x d y=0 \text { if } i \neq j .
$$

Following [6] we define $T \operatorname{Rinv}\left[L_{n}\right]=\sum_{i=1}^{n}\left\{R\left[v_{i}\right]\right\}^{-1}$. For the sums of the reciprocal eigenvalues we have the variational characterization $[5,6]$

$$
\sum_{i=1}^{n} \lambda_{i}^{-1}=\max _{L_{n}} T \operatorname{Rinv}\left[L_{n}\right] .
$$

The maximum is attained if $v_{i}=\varphi_{i} i=1, \cdots, n$ are the first $n$ eigenfunctions of Problem I. Assume that $\left(-\Delta_{z} \ln \rho\right) / 2 \rho \leqq K_{0}= \pm c^{-2}$ in $G$, 
where $K_{0}$ is any real number. In addition to Problem I we consider the auxiliary problem

II

$$
\begin{aligned}
\frac{\Delta_{w}}{(g w)} \hat{\varphi}+\hat{\lambda} \hat{\varphi} & =0 \quad \text { in } \quad C=\left\{w ;|w|<R_{a}\right\} \\
\hat{\varphi} & =0 \quad \text { on } \quad \partial C=\left\{w ;|w|=R_{a}\right\} .
\end{aligned}
$$

$g(w)$ depends on $K_{0}$ and was defined in $\S 1.1$; and $R_{a}=\sqrt{\rho(\alpha)} r_{a} / 2 c$ or $R_{a}=\sqrt{\rho(a)} r_{a}$ (cf. $\left.\S 1.1\right)$. The eigenfunctions of this problem are either of the form

$$
\widehat{\varphi}_{k}(r, \theta)=R_{0}\left(\hat{\lambda}_{k}, r\right)
$$

or

$$
\begin{gathered}
\hat{\varphi}_{k}(r, \theta)=R_{m}\left(\hat{\lambda}_{k}, r\right) \cos m \theta \quad \text { and } \quad \hat{\varphi}_{k+1}(r, \theta)=R_{m}\left(\hat{\lambda}_{k}, r\right) \sin m \theta \\
m=1,2, \cdots .
\end{gathered}
$$

In $\left(0, R_{a}\right), R_{m}\left(\hat{\lambda}_{k}, r\right)$ satisfies the differential equation

$$
\begin{gathered}
\left(r R^{\prime}\right)^{\prime}-\frac{m^{2} R}{r}+\frac{4{\hat{\lambda_{k}}}^{2} c^{2} R}{\left(1 \pm r^{2}\right)^{2}}=0 \\
\left('=\frac{d}{d r}\right)
\end{gathered}
$$

if $K_{0}= \pm c^{-2}$, and

$$
\left(r R^{\prime}\right)^{\prime}-\frac{m^{2} R}{r}+\hat{\lambda}_{k} R=0 \text { if } K_{0}=0 .
$$

The boundary conditions are

$$
R^{\prime}(0)<\infty \text { and } R\left(R_{a}\right)=0 .
$$

We shall call $m$ the order of $R$. By introducing the new variable

$$
z=\left\{\begin{array}{lll}
\left(r^{2}-1\right) /\left(1+r^{2}\right) & \text { if } \quad K_{0}>0 \\
\left(r^{2}+1\right) /\left(1-r^{2}\right) & \text { if } \quad K_{0}<0
\end{array}\right.
$$

(17) is transformed into the Legendre equation

$$
\pm \frac{d}{d z}\left[\left(z^{2}-1\right) \frac{d}{d z} y(z)\right] \mp \frac{m^{2} y(z)}{z^{2}-1}+\hat{\lambda}_{k} c^{2} y(z)=0
$$

The following result is a generalization of a theorem of Pólya-Schiffer [8]. We shall use a method of proof devised by Hersch [6].

THEoRem 1. If $(-\Delta \ln \rho) / 2 \rho \leqq K_{0}, 2 \pi-K_{0} A>0$, and $n$ is a natural number, then we have the isoperimetric inequality 


$$
\frac{1}{\lambda_{1}}+\frac{1}{\lambda_{2}}+\cdots+\frac{1}{\lambda_{n}} \geqq \frac{1}{\hat{\lambda}_{1}}+\frac{1}{\hat{\lambda}_{2}}+\cdots+\frac{1}{\hat{\lambda}_{n}}
$$

where $\hat{\lambda}_{i}$ is the $i^{\text {th }}$ eigenvalue of Problem II.

Proof. Let $\hat{\varphi}_{1}(w), \cdots, \hat{\varphi}_{n}(w)$ be the first $n$ eigenfunctions of Problem II and let $U_{1}(z), \cdots, U_{n}(z)$ be the transplanted functions $U_{i}(z)=\hat{\varphi}_{i}\left(w_{a}(z)\right)$. Because of the invariance of the Dirichlet integral under conformal transformation, we have $D_{G}\left(U_{i}, U_{j}\right)=D_{C}\left(\hat{\varphi}_{i}, \hat{\varphi}_{j}\right)=0$ if $i \neq j . \quad U_{i}(z) i=1, \cdots, n$ can therefore be used as trial functions for the variational characterization (14). Thus,

$$
\sum_{i=1}^{n} \lambda_{i}^{-1} \geqq \sum_{i=1}^{n}\left\{R\left[U_{i}\right]\right\}^{-1}=\sum_{i=1}^{n} \frac{\iint_{C} \hat{\varphi}_{i}^{2}\left|\frac{d z_{a}}{d w}\right|^{2} \rho\left(z_{a}(w)\right) d u d v}{D_{C}\left(\hat{\varphi}_{i}\right)} .
$$

Let $\hat{\varphi}_{k}(w)$ and $\hat{\varphi}_{k+1}(w)$ be two functions of the type (16). In this case

$$
\left\{R\left[U_{k}\right]\right\}^{-1}+\left\{R\left[U_{k+1}\right]\right\}^{-1}=\frac{\int_{0}^{R_{a}} \int_{0}^{2 \pi}\left(\hat{\varphi}_{k}^{2}+\hat{\varphi}_{k+1}^{2}\right) g(w)\left|\frac{d z_{a}}{d w}\right|^{2} \frac{\rho}{g} r d r d \theta}{D_{C}\left(\hat{\varphi}_{k}\right)} .
$$

We observe that

$$
\widehat{\varphi}_{k}^{2}(w)+\hat{\varphi}_{k+1}^{2}(w)=\Phi(r)
$$

is independent of $\theta$. By the Schwarz inequality,

$$
\int_{0}^{2 \pi}\left|\frac{d z_{a}}{d w}\right|^{2} \frac{\rho\left(z_{a}(w)\right)}{g(w)} r d \theta \geqq\left(\int_{0}^{2 \pi}\left|\frac{d z_{a}}{d w}\right| \sqrt{\rho} r d \theta\right)^{2} / \int_{0}^{2 \pi} g(w) r d \theta
$$

We note that for fixed $r$

$$
\int_{0}^{2 \pi}\left|\frac{d z_{a}}{d w}\right| \sqrt{\rho} r d \theta=L_{z}(t)
$$

where $L_{z}(t)$ is the length of the level line $g_{z}(z, a)=t=(1 / 2 \pi) \ln \left(R_{a} / r\right)$ in the metric of $\Sigma$. We also observe that $\int_{0}^{2 \pi} g(w) r d \theta=L_{W}^{2}(t) / 2 \pi r$, where $L_{w}(t)$ is the length of the level line $g_{w}(w, 0)=t$ with respect to the metric of $\mathscr{M}_{K_{0}}$.

In order to estimate $L_{z}^{2}(t)$, we use the following geometrical isoperimetric inequality of Alexandrow [1]: If $G$ is a domain on $\Sigma$ homeomorphic to a circle, and if $K_{G} \leqq K_{0}$, then the following relation holds between the area $A$ of $G$ and the length $L$ of the boundary $\partial G$ :

$$
L^{2} \geqq A\left(4 \pi-K_{0} A\right) \text {. }
$$

Equality holds iff $G$ is isometric to a geodesic circle on a surface of 
constant curvature $K_{0} \cdot{ }^{(1)}$ From this inequality we conclude that

$$
L_{z}^{2}(t) \geqq A_{z}(t)\left(4 \pi-K_{0} A_{z}(t)\right)=f\left(A_{z}\right) .
$$

$A_{z}(t)$ has been defined in $\S 1.2$. If $K_{0} \leqq 0$, then $f\left(A_{z}\right)$ is a monotone increasing function; if $K_{0}$ is positive then $f\left(A_{z}\right)$ is monotone increasing in the interval $\left[0,2 \pi / K_{0}\right]$. By $(26)$, (12) and our assumption on $A$, it follows that

$$
L_{z}^{2}(t) \geqq A_{w}(t)\left(4 \pi-K_{0} A_{W}(t)\right)=L_{w}^{2}(t)
$$

This implies

$$
\int_{0}^{2 \pi}\left|\frac{d z_{a}}{d w}\right|^{2} \frac{\rho\left(z_{a}(w)\right)}{g(w)} r d \theta \geqq 2 \pi r
$$

From this inequality and from (22) and (23)

$$
\left\{R\left[U_{k}\right]\right\}^{-1}+\left\{R\left[U_{k+1}\right]\right\}^{-1} \geqq 2 \hat{\lambda}_{k}^{-1} \text {. }
$$

If $\hat{\varphi}_{n}$ and $\hat{\varphi}_{n+1}$ belong to the same order $m$ [cf. (16)], we denote by $\hat{\varphi}_{n}(w)$ the function for which

$$
\left\{R\left[U_{n}\right]\right\}^{-1} \geqq \hat{\lambda}_{n}^{-1}
$$

By the same arguments as before, (27) holds also for the functions $\hat{\varphi}_{k}(w)$ of order 0 [cf. (15)]. This establishes the theorem.

Remarks. If $\rho$ is constant we obtain the theorem of Pólya-Schiffer $[8,6]$. It is easy to see that (20) is optimal if we choose a such that $R_{a}(G)=\max _{p \in G} R_{p}(G)$.

3. Generalization. Let $\Sigma^{\prime}$ be a piece of an abstract surface with the line element $d s^{2}=|z-a|^{-\omega / \pi} \nu(z)|d z|^{2}$ where $\nu(z) \in C^{2}$ and $0 \leqq \omega<2 \pi$. $\Sigma^{\prime}$ includes the regular surfaces in the usual sense which have at the point a corner of curvature $\omega$ [cf. 1]. We assume that $\left(-\Delta_{z} \ln \nu\right) / 2 \nu \leqq$ $K_{0}$. In this case we define

$$
R_{a}(G)=\left\{\begin{array}{lll}
\frac{1}{2-\omega / \pi} \sqrt{\nu(a)\left|K_{0}\right|} r_{a}(G) & \text { if } & K_{0} \neq 0 \\
\frac{2}{2-\omega / \pi} \sqrt{\nu(a)} r_{a}(G) & \text { if } & K_{0}=0 .
\end{array}\right.
$$

We consider a circular cone $\mathscr{C}_{K_{0}}$ in a three-dimensional space of constant curvature $K_{0}$ with the curvature $\omega$ at the corner [1]. It can be represented by

1 This inequality is valid for more general surfaces. A brief summary can be found in $[\mathbf{1}, \mathrm{pr} .509,514]$. 
(i) sector $0<\theta<2 \pi-\omega(\theta, r$ polar coordinates of the $w$-plane) the lines $\theta=0$ and $\theta=2 \pi-\omega$ identified, and the metric

$$
d s^{2}=\frac{4 c^{2}}{\left(1+|w|^{2}\right)^{2}}|d w|^{2} \quad\left(K_{0}=1 / c^{2}\right)
$$

(ii) sector $0<\theta<2 \pi-\omega, 0<r<1$ with the lines $\theta=0$ and $\theta=$ $2 \pi-\omega$ identified, and the metric

$$
d s^{2}=\frac{4 c^{2}}{\left(1-|w|^{2}\right)^{2}}|d w|^{2} \quad\left(K_{0}=-1 / c^{2}\right)
$$

(iii) wedge $0<\theta<2 \pi-\omega$ with the lines $\theta=0$ and $\theta=2 \pi-\omega$ identified and the metric $d s^{2}=|d w|^{2} \quad\left(K_{0}=0\right)$.

With the help of the function $\xi=w^{2 \pi /(2 \pi-\omega)}$, the sector $0<\theta<2 \pi-\omega$ is mapped into the $\xi$-plane. $g(w)$ is then transformed into $\widetilde{g}(\xi)=$ $g(w(\xi))|d w / d \xi|^{2}$ which is $\widetilde{g}(\xi)=c^{2}(2-\omega / \pi)^{2}|\xi|^{-\omega / \pi} /\left(1 \pm|\xi|^{2-\omega / \pi}\right)^{2}$ if $K_{0}=$ $\pm c^{-2}$ or $\widetilde{g}(\xi)=((2 \pi-\omega) / 2 \pi)^{2}|\xi|^{-\omega / \pi}$ if $K_{0}=0$.

ExAmple. Let $G$ be a circle with the radius $r_{0}$, the center in the origin and the metric $d s^{2}=\widetilde{g}(\xi)|d \xi|^{2}$. In this case $R_{0}(G)=r_{0}$. Let $C=\left\{\xi ;|\xi|<R_{a}(G)\right\}$ be a circle on the cone $\mathscr{C}_{K_{0}}$. The line element is then $d s^{2}=\widetilde{g}(\xi)|d \xi|^{2}$. In this metric

$$
\begin{aligned}
A_{C}= & \iint_{C} \widetilde{g}(\xi) d \xi_{1} d \eta= \begin{cases}2(2 \pi-\omega) c^{2} R_{a}^{2-\omega / \pi} /\left(1 \pm R_{a}^{2-\omega / \pi}\right) & \text { if } K_{0}= \pm c^{-2} \\
\frac{2 \pi-\omega}{2} R_{a}^{2-\omega / \pi} & \text { if } K_{0}=0\end{cases} \\
& \left(\xi=\xi_{1}+i \eta\right)
\end{aligned}
$$

is the total area of $C . \quad A=\iint_{G}|z-a|^{-\omega / \pi} \mathcal{V}(z) d x d y$ represents the total area of $G$. All properties (a), (b), (c) and (d) remain valid in this case. The proofs are the same as in $\$ 1.2$ except for (d) where we use Theorem 2 [3] instead of Corollary 2 [3].

We now consider on $\Sigma^{\prime}$ the eigenvalue problem I, and on $C \in \mathscr{C}_{K_{0}}$ the auxiliary problem II (cf. §2). By transplanting the last into the $w$-plane, it becomes equivalent to the following eigenvalue problem:

$$
\begin{gathered}
\frac{\Delta w}{g(w)} \hat{\varphi}+\hat{\lambda} \hat{\varphi}=0 \text { in }\left\{w ;|w|<R_{a}^{1-\omega / 2 \pi} \text { and } 0<\arg w<2 \pi-\omega\right\} \\
\hat{\varphi}=0 \text { on }|w|=R_{a}^{1-\omega / 2 \pi}, \\
\left.\hat{\varphi}\right|_{\theta=0}=\left.\hat{\varphi}\right|_{\theta=2 \pi-\omega} .
\end{gathered}
$$

By a separation of the variables it follows that $\hat{\varphi}(r, \theta)$ is either of 
the type $\hat{\varphi}_{k}=R_{0}\left(\hat{\lambda}_{k}, r\right)$, or else $\hat{\varphi}_{k}=R_{m}\left(\widehat{\lambda}_{k}, r\right) \cos m \theta$ and $\hat{\varphi}_{k+1}=$ $R_{m}\left(\hat{\lambda}_{k}, r\right) \sin m \theta$ with $m=2 \pi n /(2 \pi-\omega)(n=1,2, \cdots)$. In $\left(0, R_{a}^{1-\omega / 2 \pi}\right)$ $R_{m}\left(\hat{\lambda}_{k}, r\right)$ satisfies the differential equation (17) with the boundary conditions (18). In the same way as in $\S 2$ we can prove

Theorem I'. If $(-\Delta \ln \nu) / 2 \nu \leqq K_{0}$ and $2 \pi-\omega-K_{0} A>0$, then

$$
\frac{1}{\lambda_{1}}+\frac{1}{\lambda_{2}}+\cdots+\frac{1}{\lambda_{n}} \geqq \frac{1}{\hat{\lambda}_{1}}+\frac{1}{\hat{\lambda}_{2}}+\cdots+\frac{1}{\hat{\lambda}_{n}} .
$$

This inequality is valid for arbitrary $n$.

4. Bounds for the eigenvalues of plane membranes with partially free boundary. Let $G$ be a Jordan domain in the $z$-plane. Suppose that its boundary consists of three analytic arcs $\overparen{O A}, \overparen{A B}$ and $\overparen{B O}$ where $\overparen{O A}$ and $\overparen{B O}$ are concave with respect to $G$. We assume further that $\overparen{O A}$ and $\overparen{B O}$ meet in 0 at an angle $\alpha(0<\alpha \leqq \pi)$.

There exists a conformal mapping $f(z)$ from $G$ into the circular sector $0 \leqq \theta \leqq \alpha, r \leqq 1$. ( $r, \theta$ polar coordinates of the $w$-plane) such that $f(0)=0, f(A)=1, f(B)=e^{i \alpha}$ and $f^{\prime}(0)>0$ [4, p. 378]. If we put $r_{0}=\left\{f^{\prime}(0)\right\}^{-1}$, then $w(z)=r_{0} f(z)=z+a_{2} z^{2}+\cdots$. Its inverse will be called $z(w)$. We consider the following eigenvalue problem of the membrane with partially free boundary:

$$
\begin{aligned}
& \Delta_{z} \varphi+\lambda \varphi=0 \text { in } G \\
& \varphi=0 \text { on } \overparen{A B} \\
& \frac{\partial \varphi}{\partial n}=0 \text { on } \overparen{O A} \cup \overparen{B O} \text {. }
\end{aligned}
$$

These eigenvalues will be compared with the eigenvalues $\hat{\lambda}$ of the problem

$$
\text { (B) } \begin{aligned}
\Delta_{w} \hat{\varphi}+\hat{\lambda} \hat{\varphi} & =0 \quad \text { in } \quad \hat{G}=\left\{w ;|w|<r_{0} \quad \text { and } \quad 0<\arg w<\alpha\right\} \\
\hat{\varphi} & =0 \quad \text { on } \quad r=r_{0} \\
\left.\hat{\varphi}\right|_{\theta=0} & =\left.\hat{\varphi}\right|_{\theta=\alpha} .
\end{aligned}
$$

The solutions of (B) are

$$
\hat{\varphi}_{k}(r, \theta)=J_{0}\left(\sqrt{\widehat{\lambda}_{k}} r\right)
$$

or

$$
\hat{\varphi}_{k}(r, \theta)=J_{\frac{2 \pi m}{\alpha}}\left(\sqrt{\hat{\lambda}_{k}} r\right) \cos \frac{2 \pi m}{\alpha} \theta
$$

and 


$$
\hat{\varphi}_{k+1}(r, \theta)=\frac{J_{2 \pi m}}{\alpha}\left(\sqrt{\hat{\lambda}_{k}} r\right) \sin \frac{2 \pi m}{\alpha} \theta \quad m=1,2, \cdots .
$$

$J_{\beta}(r)$ is the Bessel function of order $\beta$. (B) can be interpeted as the problem of a vibrating membrane on a circular cone.

THEOREM II. For an arbitrary integer $n$ we have

$$
\frac{1}{\lambda_{1}}+\frac{1}{\lambda_{2}}+\cdots+\frac{1}{\lambda_{n}} \geqq \frac{1}{\hat{\lambda}_{1}}+\frac{1}{\hat{\lambda}_{2}}+\cdots+\frac{1}{\hat{\lambda}_{n}} \cdot
$$

Proof. Let $f(w)=f(r)$ be a function depending only on $r$. We first show that every function $F(z)=f(w(z))$ satisfies the inequality

$$
\begin{aligned}
\iint_{G} F^{2}(z) d x d y & =\int_{0}^{r_{0}} f^{2}(r) r d r \int_{\theta=0}^{\alpha}\left|\frac{d z}{d w}\right|^{2} d \theta \\
& \geqq \alpha \int_{0}^{r_{0}} f^{2}(r) r d r=\iint_{\widehat{G}} f^{2} d u d v .
\end{aligned}
$$

By the Schwarz inequality, we have

$$
\int_{0}^{\alpha}\left|\frac{d z}{d w}\right|^{2} d \theta \geqq \frac{1}{\alpha t^{2}}\left(\int_{0}^{\alpha}\left|\frac{d z}{d w}\right| t d \theta\right)^{2} .
$$

We observe that $L(t)=\int_{0}^{\alpha}|d z / d w| t d \theta$ is the length of the $\operatorname{arc} z\left(C_{t}\right)$ where $C_{t}$ is the circular arc $w=t e^{i \theta} 0 \leqq \theta \leqq \alpha$. Let $A(t)$ denote the area of the domain $z\left(\widehat{G}_{t}\right)$, where $\widehat{G}_{t}$ is the circular sector $0 \leqq r \leqq t$, $0 \leqq \theta \leqq \alpha$. Because of the concavity of the $\operatorname{arcs} \overparen{O A}$ and $\overparen{B O}$ it follows from a reflection argument and an isoperimetric inequality by Alexandrow [1] that

$$
L^{2}(t) \geqq 2 \alpha A(t) .^{2}
$$

The function $\xi=w^{2 \pi / \alpha}$ maps the sector $0 \leqq \theta \leqq \alpha$ onto the $\xi$-plane. Let $\tilde{\theta}$ and $\tilde{r}$ be the polar coordinates of the $\xi$-plane. We have

$$
\begin{aligned}
A(t) & =\int_{0}^{t} \int_{0}^{\alpha}\left|\frac{d z}{d w}\right|^{2} r d r d \theta \\
& =\left(\frac{\alpha}{2 \pi}\right)^{2} \int_{0}^{t^{2 \pi / \alpha}} \widetilde{r}^{(\alpha-2 \pi) / \pi} \widetilde{r} d \widetilde{r} \int_{0}^{2 \pi}\left|\frac{d z}{d w}(w(\xi))\right|^{2} d \tilde{\theta} \\
& =\frac{\alpha t^{2}}{2} \cdot \frac{1}{2 \pi} \int_{0}^{2 \pi}\left|\frac{d z}{d w}(w(\xi))\right|^{2} d \tilde{\theta} .
\end{aligned}
$$

Since

$\overline{2 \text { A detailed proof }}$ with more general results can be found in [2]. 


$$
\Delta_{\xi}\left|\frac{d z}{d w}(w(\xi))\right|^{2}=4 \frac{\partial}{\partial \xi} \frac{\partial}{\partial \xi}\left|\frac{d z}{d w}(w(\xi))\right|^{2} \geqq 0,
$$

it follows that

$$
\frac{1}{2 \pi} \int_{0}^{2 \pi}\left|\frac{d z}{d w}\right|^{2} d \widetilde{\theta} \geqq\left|\frac{d z}{d w}\right|_{w=0}^{2}=1
$$

and hence

$$
A(t) \geqq \frac{\alpha t^{2}}{2}
$$

(32) and (30) imply

$$
\int_{1}^{\alpha}\left|\frac{d z}{d w}\right|^{2} d \theta \geqq \alpha
$$

which proves (29).

The remaining part of the proof proceeds as in Theorem $I(\S 2)$.

We transplant the eigenfunction $\hat{\varphi}_{i}$ into the $z$-plane. $U_{i}(z)=$ $\hat{\varphi}_{i}(w(z))$ are admissible for the variational characterization (14), and we thus have

$$
\sum_{i=1}^{n} \lambda_{i}^{-1} \geqq \operatorname{Trin} v\left[L\left(U_{1}, \cdots, U_{n}\right)\right]=\sum_{i=1}^{n} \frac{\iint_{G} \hat{\varphi}_{i}^{2}\left|\frac{d z}{d w}\right|^{2} d u d v}{D_{\hat{a}}\left(\hat{\varphi}_{i}\right)} .
$$

If

$$
\hat{\varphi}_{k}(r, \theta)=J_{\frac{2 \pi m}{\alpha}}\left(\sqrt{\hat{\lambda}_{k} r}\right) \cos \frac{2 \pi m \theta}{\alpha}
$$

and

$$
\widehat{\varphi}_{k+1}(r, \theta)=J_{\frac{2 \pi m}{\alpha}}\left(\sqrt{\hat{\lambda}_{k} r}\right) \sin \frac{2 \pi m}{\alpha} \theta
$$

then (29) implies

$$
\left\{R\left[U_{k}\right]\right\}^{-1}+\left\{R\left[U_{k+1}\right]\right\}^{-1} \geqq 2 \hat{\lambda}_{k}^{-1}
$$

For functions $\hat{\varphi}_{k}$ which depends only on $r$ we have $\left\{R\left[U_{k}\right]\right\}^{-1} \geqq \hat{\lambda}_{k}$. It is always possible to choose $\hat{\varphi}_{n}(r, \theta)$ such that the last inequality remains true for $k=n$. These relations together with (34) establish the theorem.

The first eigenvalue $\hat{\lambda}_{1}$ of problem (B) is the same as the first eigenvalue $\nu_{1}$, of the problem $\Delta_{w} \widetilde{\varphi}+\nu \widetilde{\varphi}=0$ in $G, \widetilde{\varphi}=0$ on $r=r_{0}$, $\partial \tilde{\varphi} / \partial n=0$ on $\theta=0$ and $\theta=\alpha$. Theorem II and Theorem III in [2] yield the 
Corollary. If $A$ denotes the total area of $G$ and $j_{0}=2,4048 \ldots$ is the first zero of the Bessel function $J_{0}(r)$, then

$$
\frac{\alpha}{2 A} j_{0}^{2} \leqq \lambda_{1} \leqq\left(\frac{j_{0}}{r_{0}}\right)^{2}
$$

Equality holds in both cases if and only if $G$ is a circular sector.

The right-hand side of (36) is a generalization of an inequality by pólya and Szegö [8]. The following charaterization of $r_{0}$ is based on the one indicated in [8] for the conformal radius. Let $\mu\left(\overparen{A B}, \Gamma_{\varepsilon}\right)$ be the modulus of the domain $G_{\varepsilon} \subseteq G$ bounded by $\overparen{A B}, \overparen{B O}, \overparen{O A}$ and $\Gamma_{\varepsilon}=\{z ;|z|=\varepsilon\}$. It is defined as $\mu\left(\overparen{A B}, \Gamma_{\varepsilon}\right)=1 / D(h)$ where $\Delta h=0$ in $G_{\varepsilon}, h=1$ on $\Gamma_{\varepsilon}$ and $h=0$ on $\overparen{A B}$. An easy computation (cf. $\S 1$ (c)) yields

$$
r_{0}=\lim _{\varepsilon \rightarrow 0} \varepsilon e^{\alpha \mu\left(A B, \Gamma_{\varepsilon}\right)}
$$

Let $D$ denote the shortest distance from the arc $\overparen{A B}$ to the origin 0 . By (37) and the monotonicity of $\mu\left(\overparen{A B}, \Gamma_{\varepsilon}\right)$ it follows that $D \leqq r_{0}$. This inequality together with the corollary implies $\lambda_{1} \leqq\left(j_{0} / D\right)^{2}$.

\section{REFERENCES}

1. A. D. Alexandrow, Die innere Geometrie der konvexen Flächen, Berlin, 1955.

2. C. Bandle, Extremaleigenschaften von Kreissektoren und Halbkugeln, To appear in Comment. Math. Helv., 46 (1971), 356-380.

3. - Konstruktion isoperimetrischer Ungleichungen der Mathematischen Physik aus solchen der Geometrie, Comment. Math. Helv., 46 (1971), 182-213.

4. Behnke-Sommer, Theorie der analytischen Functione einer komplexen Veränderlichen, Berlin Göttingen, Heidelberg 1955.

5. R. Courant, and D. Hilbert, Methods of Mathematical Physics, New York, 1965, vol. 1.

6. J. Hersch, On symmetric memberanes and conformal radius: Some complements to Pólya's and Szegö's Inequalities, Arch. Rat. Mech. Anal., 20 (1965), 378-395.

7. R. Osserman, On the inequality $\Delta u \geqq f(u)$, Pacific J. Math., 7 (1957), 1641-1647.

8. G. Pólya, and M. Schiffer, Convexity of functionals by transplantation, J. d'Anal. Math., 3 (2 partie) (1953/54) 245/345.

9. G. Pólya, and G. Szegö, Aufgaben und Lehrsätze aus der Analysis II, Berlin, Springer-Verlag, 1964.

10. - Isoperimetric Inequalities in Mathematical Physics, Princeton University Press, 1951.

Received June 8, 1971. The work was supported by NSF Grant GU-2056

Carnegie-Mellon University

PRESENT ADRESS:

STANFORD UNIVESITY 



\section{PACIFIC JOURNAL OF MATHEMATICS}

\section{EDITORS}

\section{H. SAMELSON}

Stanford University

Stanford, California 94305

\section{R. HOBBY}

University of Washington

Seattle, Washington 98105

\section{J. DugundjI}

Department of Mathematics University of Southern California Los Angeles, California 90007

RICHARD ARENS

University of California

Los Angeles, California 90024

\section{ASSOCIATE EDITORS}
E. F. BECKENBACH
B. H. NeumanN
F. WOLF
K. YOSHIDA

\section{SUPPORTING INSTITUTIONS}

\author{
UNIVERSITY OF BRITISH COLUMBIA \\ CALIFORNIA INSTITUTE OF TECHNOLOGY \\ UNIVERSITY OF CALIFORNIA \\ MONTANA STATE UNIVERSITY \\ UNIVERSITY OF NEVADA \\ NEW MEXICO STATE UNIVERSITY \\ OREGON STATE UNIVERSITY \\ UNIVERSITY OF OREGON \\ OSAKA UNIVERSITY
}

\author{
UNIVERSITY OF SOUTHERN CALIFORNIA \\ STANFORD UNIVERSITY \\ UNIVERSITY OF TOKYO \\ UNIVERSITY OF UTAH \\ WASHINGTON STATE UNIVERSITY \\ UNIVERSITY OF WASHINGTON \\ AMERICAN MATHEMATICAL SOCIETY \\ NAVAL WEAPONS CENTER
}

The Supporting Institutions listed above contribute to the cost of publication of this Journal, but they are not owners or publishers and have no responsibility for its content or policies.

Mathematical papers intended for publication in the Pacific Journal of Mathematics should be in typed form or offset-reproduced, (not dittoed), double spaced with large margins. Underline Greek letters in red, German in green, and script in blue. The first paragraph or two must be capable of being used separately as a synopsis of the entire paper. The editorial "we" must not be used in the synopsis, and items of the bibliography should not be cited there unless absolutely necessary, in which case they must be identified by author and Journal, rather than by item number. Manuscripts, in duplicate if possible, may be sent to any one of the four editors. Please classify according to the scheme of Math. Rev. Index to Vol. 39. All other communications to the editors should be addressed to the managing editor, Richard Arens, University of California, Los Angeles, California, 90024.

50 reprints are provided free for each article; additional copies may be obtained at cost in multiples of 50 .

The Pacific Journal of Mathematics is published monthly. Effective with Volume 16 the price per volume (3 numbers) is $\$ 8.00$; single issues, $\$ 3.00$. Special price for current issues to individual faculty members of supporting institutions and to individual members of the American Mathematical Society: $\$ 4.00$ per volume; single issues $\$ 1.50$. Back numbers are available.

Subscriptions, orders for back numbers, and changes of address should be sent to Pacific Journal of Mathematics, 103 Highland Boulevard, Berkeley, California, 94708.

PUBLISHED BY PACIFIC JOURNAL OF MATHEMATICS, A NON-PROFIT CORPORATION

Printed at Kokusai Bunken Insatsusha (International Academic Printing Co., Ltd.), 270, 3-chome Totsuka-cho, Shinjuku-ku, Tokyo 160, Japan. 


\section{Pacific Journal of Mathematics}

\section{Vol. 42, No. $3 \quad$ March, 1972}

Catherine Bandle, Extensions of an inequality by Pólya and Schiffer for vibrating membranes ................................ 543

S. J. Bernau, Topologies on structure spaces of lattice groups.......... 557

Woodrow Wilson Bledsoe and Charles Edward Wilks, On Borel product measures .......................................

Eggert Briem and Murali Rao, Normpreserving extensions in subspaces of

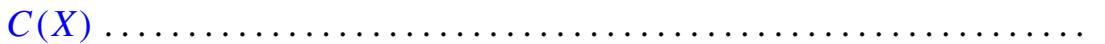

Alan Seymour Cover, Generalized continuation.................. 589

Larry Jean Cummings, Transformations of symmetric tensors .......... 603

Peter Michael Curran, Cohomology of finitely presented groups .......... 615

James B. Derr and N. P. Mukherjee, Generalized quasicenter and

hyperquasicenter of a finite group ...................... 621

Erik Maurice Ellentuck, Universal cosimple isols .................. 629

Benny Dan Evans, Boundary respecting maps of 3-mainfolds .......... 639

David F. Fraser, A probabilistic method for the rate of convergence to the

Dirichlet problem .................................. 657

Raymond Taylor Hoobler, Cohomology in the finite topology and Brauer

groups ..................................... 667

Louis Roberts Hunt, Locally holomorphic sets and the Levi form ........ 681

B. T. Y. Kwee, On absolute de la Vallée Poussin summability............ 689

Gérard Lallement, On nilpotency and residual finiteness in semigroups .... 693

George Edward Lang, Evaluation subgroups of factor spaces........... 701

Andy R. Magid, A separably closed ring with nonzero torsion pic ....... 711

Billy E. Rhoades, Commutants of some Hausdorff matrices ............. 715

Maxwell Alexander Rosenlicht, Canonical forms for local derivations . . . . 721

Cedric Felix Schubert, On a conjecture of L. B. Page ................ 733

Reinhard Schultz, Composition constructions on diffeomorphisms of $S^{p} \times S^{q}$

J. P. Singhal and H. M. (Hari Mohan) Srivastava, A class of bilateral generating functions for certain classical polynomials ....

Richard Alan Slocum, Using brick partitionings to establish conditions which insure that a Peano continuum is a 2-cell, a 2-sphere or an annulus...

James F. Smith, The p-classes of an $H^{*}$-algebra ...

Jack Williamson, Meromorphic functions with negative zeros and positive

poles and a theorem of Teichmuller ................. 